\title{
On the EVM computation of arbitrary clipped multi-carrier signals
}

\author{
Igal Kotzer ${ }^{*}$ and Simon Litsyn
}

\begin{abstract}
A common figure of merit in multi-carrier systems is the error vector magnitude (EVM). A method for EVM computation of a multi-carrier signal without any underlying model (e.g., the Gaussianity assumption) was proposed in a previous work of the authors. However, it addressed only the case of identical constellations and power loadings in all tones. In practice, however, the constellation and power loading may vary among the tones (e.g., boosted pilots, waterfilling and zero guard bands). Here the earlier approach is generalized in such a way that it is able to accommodate for an accurate analytical EVM computation in the cases of power loading and different constellations for different tones. Moreover, the derivation is valid for a general magnitude clipping function, so that any realistic clipper can be plugged in.
\end{abstract}

\section{Introduction}

The use of multi-carrier (MC) communication schemes (e.g., OFDM, DMT, etc.) is very common nowadays due to its ability to cope well with channel interference while keeping the receiver complexity low, the ease of spectral mask shaping and high spectral efficiency. However, one of MC scheme's greatest drawbacks is the high peak-toaverage power ratio (PAPR) caused by various degrees of coherent summation in the signal generation using IFFT [1]. Thus, systems utilizing MC communications must work with a large back-off in the high-power amplifier (HPA), which reduces both the efficiency of the HPA and the average power transmitted, or risk clipping. Based on the understanding that clipping is a nonlinear operation causing both in-band and out-of-band spectral noise and thus is an undesirable operation, methods for reducing the PAPR were devised. For a survey see [1-4]. Most of the power reduction methods are either statistical in naturethat is they do not guarantee PAPR limits, or iterative-in which required PAPR limits are easier to meet at the expense of computational complexity. Hence, while it is understood that the amount of clipping should be minimized, due to practical system limitations clipping cannot be entirely eliminated, but rather be set on a compromise level. Therefore, evaluating the performance of MC systems with clipping becomes relevant.

\footnotetext{
* Correspondence: igalk@eng.tau.ac.il

School of Electrical Engineering - Systems, Tel Aviv University, Israel
}

Two prominent criteria for evaluating the performance of a MC system are its capacity [5-7], and the system's error probability $[8,9]$. However, in engineering practice, the most popular measure is the error vector magnitude (EVM). The EVM is a figure of merit for inband distortion, which does not only quantifies the distortion but in some cases can attribute impairments to various system components [10]. Due to its popularity and troubleshooting capabilities, the EVM has become a mandatory part of a few communication standards, e.g. [[11], Tables 165, 172].

In [12] the authors express the EVM of an OFDM signal impaired by clipping without relying on the Gaussianity assumption and show that the EVM can be expressed with an arbitrary precision as a power series of the number of tones with constellation-dependent coefficients. It is also shown that for some specific constellations the EVM can be calculated via easy to use expressions without the need for a power series expansion. However, these computations fit the case of MC signals with an identical constellation for all tones and no power loading. Yet, real world signal utilize both different constellations for different tones and power loading. Some of the tones are zeroed due to spectral mask considerations, while some tones are boosted (e.g., pilot tones) to allow better channel tracking. A waterfilling solution in high SNR MIMO OFDM or in DMT also requires adjusting power and thus constellation to each tone individually. In this paper we address the issue of various constellations and power loading in the 
MC signal as well as the effect of an arbitrary magnitude clipping response by giving an analytical expression in the form of a power series for computing the EVM of the generalized case.

Analysis of clipped signals usually relies on the Gaussianity assumption [5]. However, this assumption is not always valid, especially for a mix of BPSK and QAM constellations. Hence, in order to evaluate the performance of such systems one must resort to numerical evaluations. This work allows to accurately compute the EVM of clipped signals for any constellation mixture and clipping function without the need to redo the numerical evaluation for each desired scenario.

The paper is organized as follows. In Section 2 the system model used in this work is introduced. Theorem 3.1 in Section 3 presents the main result of this work. In Section 4 we present simulation results and compare them to the theoretical results about EVM derived in this work.

\section{System model}

The system model discussed in this work is depicted in Figure 1. The vector $\boldsymbol{a}=\left[a_{0}, a_{1}, \ldots, a_{N-1}\right]^{T}$ denotes the $N$ data symbols vector in the form of constellation points, e.g., $\boldsymbol{a} \in\{+1,-1\}^{N}$ for BPSK. The vector $\boldsymbol{x}=\left[x_{0}, x_{1}, \ldots\right.$, $\left.x_{N-1}\right]^{T}$ denotes the time domain discrete time signal and is obtained by applying the inverse discrete Fourier transform on $\boldsymbol{a}$ :

$$
x_{n}=\frac{1}{\sqrt{N}} \sum_{k=0}^{N-1} a_{k} e^{\frac{i 2 \pi k n}{N}}, \quad 0 \leq n \leq N-1 .
$$

The vector $\boldsymbol{y}$ denotes the vector $\boldsymbol{x}$ after clipping operation. Two clipping functions we will specifically address are the SSPA clipper [13]:

$$
y_{n}=\frac{x_{n}}{\left[1+\left(\frac{\left|x_{n}\right|}{c}\right)^{2 p}\right]^{1 / 2 p}}
$$

and the soft clipper (which is a special case of the SSPA clipper for $p \gg 1$ ):

$$
y_{n}=\left\{\begin{array}{ll}
x_{n} & \left|x_{n}\right|<c \\
c e^{i \varangle x_{n}} & \left|x_{n}\right| \geq c
\end{array},\right.
$$

where $c$ is the clipping level. The noise vector $\boldsymbol{w}$ denotes an AWGN with variance $\sigma_{w}^{2}$ and is independent of $\boldsymbol{a} . \hat{x}$ is the noisy clipped discrete time domain signal

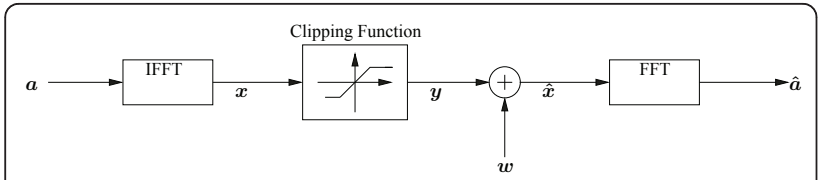

Figure 1 Baseband discrete time AWGN channel model. and $\hat{a}$ is the data symbols vector reconstructed from the clipped and noisy signal. For this system we define the EVM as

$$
\mathrm{EVM} \triangleq \sqrt{\frac{\mathbb{E}\left\{|\hat{\boldsymbol{a}}-\boldsymbol{a}|^{2}\right\}}{N \cdot \mathbb{E}\left\{\left|a_{k}\right|^{2}\right\}}}
$$

Assuming the constellation energy $\mathbb{E}\left\{\left|a_{k}\right|^{2}\right\}$ is known and the noise variance is known, we need to calculate the error power $\mathbb{E}\left\{|\hat{\boldsymbol{a}}-\boldsymbol{a}|^{2}\right\}$ to be able to evaluate the EVM. By virtue of Parseval's theorem, we have

$$
\sum_{n=0}^{N-1}\left|x_{n}\right|^{2}=\sum_{k=0}^{N-1}\left|a_{k}\right|^{2}
$$

Hence, it immediately follows that

$$
\mathbb{E}\left\{|\hat{\boldsymbol{a}}-\boldsymbol{a}|^{2}\right\}=\mathbb{E}\left\{|\hat{\boldsymbol{x}}-\boldsymbol{x}|^{2}\right\}=\sum_{n=0}^{N-1} \mathbb{E}\left\{\left|y_{n}-x_{n}\right|^{2}\right\}+N \sigma_{w}^{2} .
$$

The EVM contribution due to clipping can thus be calculated by computing the quantity $\mathbb{E}\left\{\left|y_{n}-x_{n}\right|^{2}\right\}$ for every $0 \leq n \leq N-1$. Obviously, for scenaria with large channel noise we can allow more signal distortion due to clipping as long as it is negligible relative to the channel noise.

\section{EVM computation}

In this section we present the main result. Let $f\left(\left|x_{n}\right|\right)=f$ $(r)$ be the energy of the clipped portion of the sample $x_{n}$, and let us decompose the symbols vector $\boldsymbol{a}$ of length $N$ into three groups:

- $N_{B}$ groups of BPSK symbols. The symbols of each group, of size $N_{r}$, are drawn from a constellation with energy $E_{r}=b_{r}^{2}$ for $1 \leq r \leq N_{B}$.

- $N_{Q}$ groups of QAM symbols. The symbols of each group, of size $N_{s}$, are drawn from a constellation of size $M s$, with constellation coefficients $v_{s, l m}$ (which are the series expansion coefficients of a function of the constellation -see Appendix A for details.) and energy $E_{s}=q_{s}^{2}$ for $1 \leq s \leq N_{Q}$. For example, for QPSK of the form $a_{k_{\mathcal{R}}}, a_{k_{I}} \in( \pm 1 / \sqrt{2})$ we have $v_{s, 11}$ $=-1 / 4, v_{s, 22}=-1 / 64$, and for 16QAM of the form $a_{k_{I}} \in( \pm 1, \pm 3) / \sqrt{10}, a_{k_{I}} \in( \pm 1, \pm 3) / \sqrt{10}$ we have $v_{s, 11}=-1 / 4$ and $v_{s, 22}=-17 / 1600$.

- $N_{Z}$ zero tones.

Clearly $N_{B}+N_{Q}+N_{Z}=N$. Then, the following quantities are defined:

$$
\mu_{1}=\left[\sum_{s=1}^{N_{Q}} \frac{q_{s}^{2} N_{s} v_{s, 11}}{N}-\sum_{r=1}^{N_{B}} \frac{b_{r}^{2} N_{r}}{4 N}\right]
$$


and

$$
\mu_{2}=\left[\sum_{s=1}^{N_{Q}} \frac{q_{s}^{4} N_{s} v_{s, 22}}{N^{2}}-\sum_{r=1}^{N_{B}} \frac{b_{r}^{4} N_{r}}{32 N^{2}}\right] .
$$

In addition, let $\tilde{\mu}_{1}=N \mu_{1}$ and $\tilde{\mu}_{2}=N^{2} \mu_{2}$.

Theorem 3.1. The term $\sum_{n=0}^{N-1} \mathbb{E}\left\{\left|y_{n}-x_{n}\right|^{2}\right\}$ in (6) can be calculated as follows:

$$
\sum_{n=0}^{N-1} \mathbb{E}\left\{\left|y_{n}-x_{n}\right|^{2}\right\}=N^{2} \sum_{q=0}^{\infty} m_{q}(c) N^{q},
$$

where $m_{q}(c)$ depend on the clipping level, the constellations, power loading and symbol length. In particular, $m_{0}$ (c) and $m_{1}(c)$ can be calculated as follows:

$$
\begin{aligned}
& m_{0}(c)=-\int_{0}^{\infty} r f(r) \exp \left(\frac{r^{2}}{4 \mu_{1}}\right)\left(\frac{1}{2 \tilde{\mu}_{1}}+\frac{\tilde{\mu}_{2}}{\tilde{\mu}_{1}^{3}}\right) d r \\
& m_{1}(c)=-\int_{0}^{\infty} r f(r) \exp \left(\frac{r^{2}}{4 \mu_{1}}\right)\left(\frac{\tilde{\mu}_{2}}{2 \tilde{\mu}_{1}^{4}} r^{2}+\frac{\tilde{\mu}_{2}}{32 \tilde{\mu}_{1}^{5}} r^{4}\right) d r .
\end{aligned}
$$

Proof. See Appendix A. $\square$

\section{Simulation results and discussion}

\subsection{The Gaussian approximation}

A common method for analyzing the EVM of an OFDM signal uses the central limit theorem (CLT). By invoking the CLT $x_{n}$ are assumed to be distributed complex normally, i.e. $x_{n} \sim \operatorname{CN}\left(0, \sigma^{2}\right)$, and thus $\left|x_{n}\right| \sim \operatorname{Rayleigh}(\sigma)$. Hence, the EVM can be computed in a straightforward method:

$$
\sum_{n=0}^{N-1} \mathbb{E}\left\{\left|y_{n}-x_{n}\right|^{2}\right\}=N \int_{0}^{\infty} f(r) \frac{r}{\sigma^{2}} \exp \left\{-\frac{r^{2}}{2 \sigma^{2}}\right\} d r,(
$$

where $f(r)=f\left(\left|x_{n}\right|\right)$ is the clipping function in polar coordinates. In this work, when the results are compared to the Gaussian approximation it is assumed that $\sigma^{2}=1$.

\subsection{Simulation results}

In the following examples two cases of magnitude clipping functions are considered. The SSPA clipper, for which

$$
f\left(\left|x_{n}\right|\right)=f(r)=\left[\frac{r}{\left(1+(r / c)^{2 p}\right)^{1 / 2 p}}-r\right]^{2},
$$

and the soft clipper, for which $f\left(\left|x_{n}\right|\right)=f(r)=(r-c)_{+}^{2}$, where the operation ()$_{+}$denotes taking only the positive part. The soft clipper is a special case of the SSPA clipper for $p \rightarrow \infty$, which can be practically achieved with $p>$ 100. In the following simulations $p=200$ was chosen.
Figure 2a demonstrates the EVM versus clipping level for the mixture of 64 BPSK modulated tones, 320 16QAM modulated tones and 128 zero tones, all randomly spread across the symbol. That is, $N_{B}=1, N_{r=1}=64, N_{Q}=1$, $N_{s=1}=320, v_{1,11}=-1 / 4$ and $v_{1,22}=-17 / 1600$. In this figure all constellation energies are normalized to unity (i.e. $b_{r}=q_{s}=1$ ). Figure $2 \mathrm{~b}$ demonstrates the EVM versus clipping level for the mixture of 128 BPSK modulated tones with constellation energy boosted by $3 \mathrm{~dB}, 128$ QPSK modulated tones and 256 16QAM modulated tones (the two latter constellations are with unity constellation energy). Namely, for Figure $2 b$, the simulation parameters are $N_{B}=1, N_{r=1}=128, b_{r}=\sqrt{2}$, and $N_{Q}=2$ with $N_{s=1}=128, v_{1,11}=-1 / 4, v_{1,22}=-1 / 64, \mathrm{q}_{1}=1$ and $N_{s=2}=256, v_{2,11}=-1 / 4, v_{2,22}=-17 / 1600, q_{2}=1$.

It can be clearly seen that as the mixture becomes more diverse in tone constellations and power loading, the mixed signal's EVM diverges from the Gaussian model. Additionally, as can be expected, the less linear the clipping function, the higher the EVM is. It can be also seen that the analytical computation coincides perfectly with the simulation.

\section{Summary}

In this paper we present a method for computing the EVM of a MC signal with power loading and various constellations on various tones that is impaired by clipping. This computation does not rely on any underlying model for the signal (such as the Gaussianity assumption), making it accurate for any mixture of tone constellations and power loading. A comparison between the simulated and theoretical EVM results shows a perfect match between the two. The main result of this work can be also used with any arbitrary magnitude clipping function for achieving more realistic results for practical uses.

\section{Appendix A Proof of the EVM computation equation}

We define the energy of the clipped portion of the signal as $f\left(x_{n}\right)=f\left(x_{n_{\mathfrak{R}}}, x_{n_{I}}\right)=\left|y_{n}-x_{n}\right|^{2}$. Any clipping function can be represented as a superposition of its effect on the signal's magnitude (AM/AM) and its effect on the signal's phase (AM/PM). The AM/AM function can be further represented in terms of $\left|x_{n}\right|$. Thus, $f$ can be defined as $f\left(\sqrt{x_{n_{R}}^{2}+x_{n_{I}}^{2}}\right)=f(r)$, where $r=\sqrt{x_{n_{R}}^{2}+x_{n_{I}}^{2}}$ is the polar coordinates representation. We wish to calculate $\mathbb{E}\left\{f\left(x_{n_{\mathcal{R}}}+x_{n_{I}}\right)\right\}$ any $0 \leq n \leq N-1$. We start by representing $f\left(x_{\mathcal{R}}, x_{I}\right)$ by its inverse Fourier transform:

$$
f\left(x_{\mathcal{R}}, x_{I}\right)=\frac{1}{2 \pi} \iint_{-\infty}^{\infty} \widehat{f}\left(\omega_{1}, \omega_{2}\right) e^{i\left(\omega_{1} x_{\mathcal{R}}+\omega_{2} x_{I}\right)} d \omega_{1} d \omega_{2},
$$




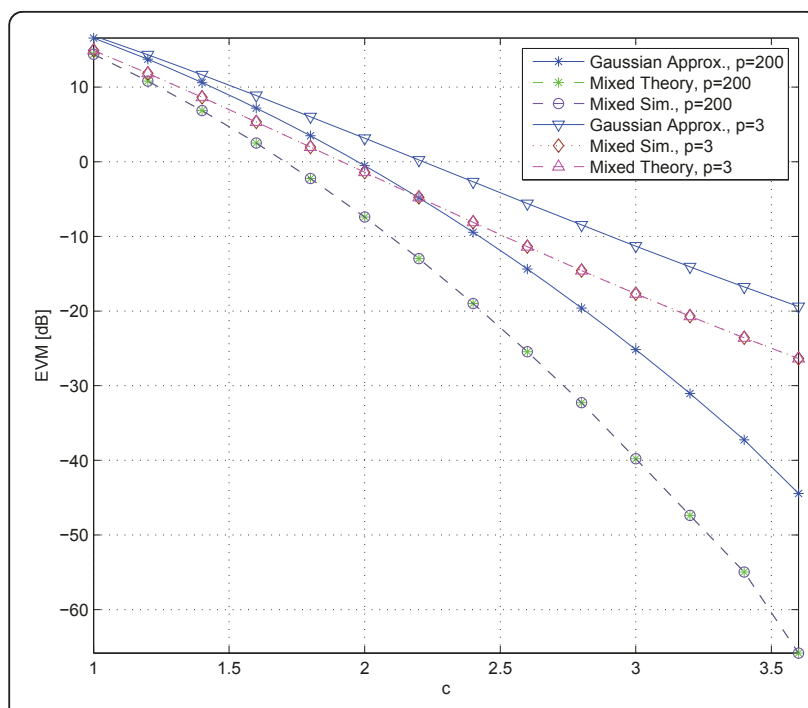

(a)

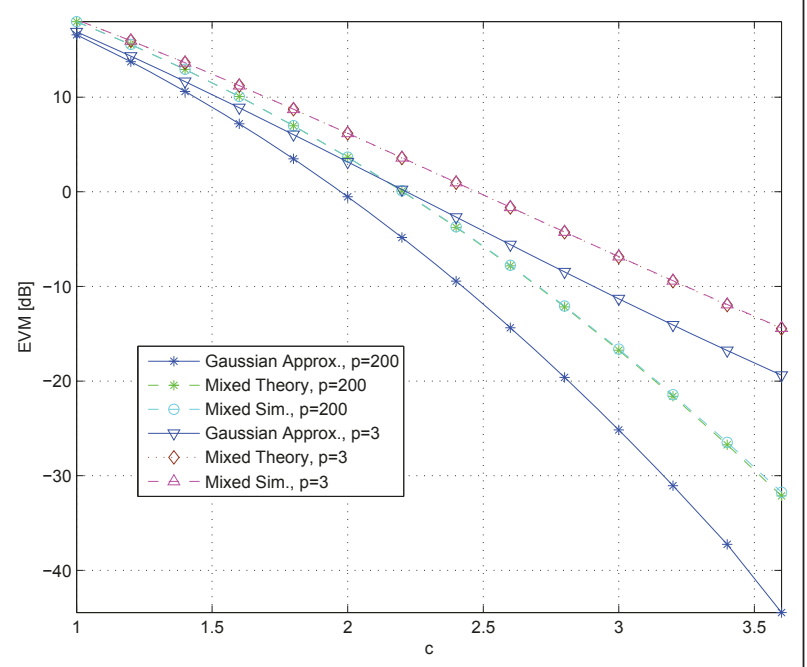

(b)

Figure 2 Simulated and theoretical EVM versus clipping level for two magnitude clipping functions. (a) Mixture of BPSK, 16QAM and zero tones. (b) Mixture of 3dB Boosted BPSK, QPSK and 16QAM tones.

where $\hat{f}\left(\omega_{1}, \omega_{2}\right)$ is the Fourier transform of $f\left(x_{\mathscr{R}}, x_{I}\right)$ :

$$
\begin{aligned}
\hat{f}\left(\omega_{1}, \omega_{2}\right) & =\frac{1}{2 \pi} \iint_{-\infty}^{\infty} f\left(x_{\mathcal{R}}, x_{I}\right) e^{-i\left(\omega_{1} x_{\mathcal{R}}+\omega_{2} x_{I}\right)} d x_{\mathcal{R}} d x_{I} \\
& =\int_{0}^{\infty} r f(r) \frac{1}{2 \pi} \int_{0}^{2 \pi} e^{-i r\left(\omega_{1} \cos (\theta)+\omega_{2} \sin (\theta)\right)} d \theta d r \\
= & \int_{0}^{\infty} r f(r) J_{0}\left(r \sqrt{\omega_{1}^{2}+\omega_{2}^{2}}\right) d r
\end{aligned}
$$

where $J_{0}$ is the Bessel function of the first kind and zeroth order. Furthermore, $x_{n}$ can be written explicitly as a sum of its real and imaginary parts as follows:

$$
\begin{aligned}
& x_{n_{\mathcal{R}}}=\frac{1}{\sqrt{N}} \sum_{k=0}^{N-1}\left\{a_{k_{\mathcal{R}}} \cos \left(\frac{2 \pi k n}{N}\right)-a_{k_{I}} \sin \left(\frac{2 \pi k n}{N}\right)\right\}, \\
& x_{n_{I}}=\frac{1}{\sqrt{N}} \sum_{k=0}^{N-1}\left\{a_{k_{\mathcal{R}}} \sin \left(\frac{2 \pi k n}{N}\right)+a_{k_{I}} \cos \left(\frac{2 \pi k n}{N}\right)\right\} .
\end{aligned}
$$

Thus, we can substitute (16) into(13) and rewrite $f\left(x_{R}, x_{I}\right)$ as

$$
\begin{aligned}
f\left(x_{n_{R^{\prime}}}, x_{n_{1}}\right)= & \frac{1}{2 \pi} \iint_{-\infty}^{\infty} \hat{f}\left(\omega_{1}, \omega_{2}\right) \exp \left\{\frac { i } { \sqrt { N } } \left[\omega_{1} \sum_{k=0}^{N-1}\left(a_{k_{\ell}} \cos \left(\frac{2 \pi k n}{N}\right)-a_{k_{1}} \sin \left(\frac{2 \pi k n}{N}\right)\right)\right.\right. \\
& \left.\left.+\omega_{2} \sum_{k=0}^{N-1}\left(a_{k_{\ell}} \sin \left(\frac{2 \pi k n}{N}\right)+a_{k_{l}} \cos \left(\frac{2 \pi k n}{N}\right)\right)\right]\right\} d \omega_{1} d \omega_{2} \\
= & \frac{1}{2 \pi} \iint_{-\infty}^{\infty} \hat{f}\left(\omega_{1}, \omega_{2}\right) \exp \left\{\frac { i } { \sqrt { N } } \sum _ { k = 0 } ^ { N - 1 } \left[a_{k_{k}}\left[\omega_{1} \cos \left(\frac{2 \pi k n}{N}\right)+\omega_{2} \sin \left(\frac{2 \pi k n}{N}\right)\right]\right.\right. \\
& \left.\left.+a_{k_{1}}\left[-\omega_{1} \sin \left(\frac{2 \pi k n}{N}\right)+\omega_{2} \cos \left(\frac{2 \pi k n}{N}\right)\right]\right]\right\} d \omega_{1} d \omega_{2} .
\end{aligned}
$$

Denoting

$$
\phi_{k}(\alpha, \beta)=\mathbb{E}\left(e^{i\left(\alpha a_{k_{\mathcal{R}}}+\beta a_{k_{I}}\right.}\right),
$$

and using the fact that $a_{k}$ are independent, we can write:

$$
\begin{aligned}
& \mathrm{E}\left\{f\left(x_{n_{n^{\prime}}}, x_{n_{l}}\right)\right\}=\frac{1}{2 \pi} \iint_{\left(\omega_{1}, \omega_{2}\right) \in \mathbb{R}^{2}} \hat{f}\left(\omega_{1}, \omega_{2}\right) \\
& \quad \prod_{k=0}^{N-1} \phi_{k}\left\{\frac{\omega_{1} \cos \left(\frac{2 \pi k n}{N}\right)+\omega_{2} \sin \left(\frac{2 \pi k n}{N}\right)}{\sqrt{N}}, \frac{-\omega_{1} \sin \left(\frac{2 \pi k n}{N}\right)+\omega_{2} \cos \left(\frac{2 \pi k n}{N}\right)}{\sqrt{N}}\right\} d \omega_{1} d \omega_{2} .
\end{aligned}
$$

Therefore, according to(15)

$$
\begin{aligned}
& \mathbb{E}\left\{f\left(x_{n_{n^{\prime}},} x_{n_{l}}\right)\right\}=\frac{1}{2 \pi} \int_{0}^{\infty} r f(r) \iint_{\left(\omega_{1}, \omega_{2}\right) \in \mathbb{R}^{2}} J_{0}\left(r \sqrt{\omega_{1}^{2}+\omega_{2}^{2}}\right) \\
& \cdot \prod_{k=0}^{N-1} \phi_{k}\left\{\frac{\omega_{1} \cos \left(\frac{2 \pi k n}{N}\right)+\omega_{2} \sin \left(\frac{2 \pi k n}{N}\right)}{\sqrt{N}}, \frac{-\omega_{1} \sin \left(\frac{2 \pi k n}{N}\right)+\omega_{2} \cos \left(\frac{2 \pi k n}{N}\right)}{\sqrt{N}}\right\} d \omega_{1} d \omega_{2} d r .
\end{aligned}
$$

We now proceed to calculate the term $\prod_{k=0}^{N-1} \phi_{k}$ of (20) by expanding to a power series the term $\sum_{k=0}^{N-1} \ln \phi_{k}$ and then taking the exponent of the series. Unlike [12], if $a_{k}$ are not identically distributed then $\varphi_{k}$ must be computed for every $k$, or alternatively for every type of constellation and then combined together. We rewrite the arguments of $\varphi_{k}$ as follows:

$$
\begin{aligned}
\mathbb{E}\left\{f\left(x_{n_{\mathcal{R}^{\prime}}} x_{n_{I}}\right)\right\}= & \frac{1}{2 \pi} \int_{0}^{\infty} r f(r) \iint_{\left(\omega_{1}, \omega_{2}\right) \in \mathbb{R}^{2}} J_{0}\left(r \sqrt{\omega_{1}^{2}+\omega_{2}^{2}}\right) \prod_{k=0}^{N-1} \\
& \cdot \phi_{k}\left\{\Re\left(\frac{\zeta \omega^{-k n}}{\sqrt{N}}\right), \Im\left(\frac{\zeta \omega^{-k n}}{\sqrt{N}}\right)\right\} d \omega_{1} d \omega_{2} d r,
\end{aligned}
$$

where $\zeta=\omega_{1}+i \omega_{2}, \bar{\zeta}$ is the complex conjugate of $\zeta$ and $\omega=\exp (2 \pi i / N)$. Denoting $z=\frac{\zeta \omega^{-k n}}{\sqrt{N}}$ we can write $\varphi_{k}$ as follows:

$$
\phi_{k}(z)=\phi_{k}(\Re z, \Im z)=\mathbb{E} \exp \left\{i\left(\Re z \cdot a_{k_{\mathscr{R}}}+\Im z \cdot a_{k_{I}}\right)\right\}
$$


as in(18). We expand In $\varphi_{k}$ as a power series:

$$
\ln \phi_{k}(z)=\ln \phi_{k}\left(\frac{\zeta \omega^{-k n}}{\sqrt{N}}\right)=\sum_{l, m \geq 0} v_{l m}^{(k)} z^{l} \bar{z}^{m},
$$

where we choose the representation $\phi_{k}(z)=\phi_{k}(z, \bar{z})=\mathbb{E} \exp \left\{i\left(\left(\frac{z+\bar{z}}{2}\right) a_{k_{\mathcal{R}}}+\left(\frac{z-\bar{z}}{2 i}\right) a_{k_{I}}\right)\right\} . \quad$ We further assume that the data symbols vector $\boldsymbol{a}$ contains $p \leq N$ distinct groups of $N_{\iota}, 1 \leq \imath \leq p$ symbols, where each group is drawn from the set of BPSK, QAM or zero constellation points with an average constellation energy of $E_{\iota}, 1 \leq \iota \leq p$. That is, groups of symbols are distinguished by the constellation type and by the average constellation energy. Hence, we have

$$
\sum_{k=0}^{N-1} \ln \phi_{k}\left(\frac{\zeta \omega^{-k n}}{\sqrt{N}}\right)=\sum_{k=0}^{N_{1}-1} \ln \phi_{1}\left(\frac{\zeta \omega^{-k n}}{\sqrt{N}}\right)+\sum_{k=0}^{N_{2}-1} \ln \phi_{2}\left(\frac{\zeta \omega^{-k n}}{\sqrt{N}}\right)+\cdots+\sum_{k=0}^{N_{p}-1} \ln \phi_{p}\left(\frac{\zeta \omega^{-k n}}{\sqrt{N}}\right) .
$$

Now we proceed to compute In $\varphi_{k}$ for each type of constellation:

- $\mathbf{a}_{\mathbf{k}}=\mathbf{0}$ : This option is usually used to generate guard bands [11]. For this option $\varphi_{k}=1$, and hence in $\varphi_{k}=0$.

- BPSK $\left(\mathbf{a}_{\mathbf{k}}= \pm \mathbf{b}=\mathbf{b} \cdot\{ \pm \mathbf{1}\}\right)$ : First, it is noted that $a_{k}$ are drawn from a BPSK constellation with energy $E_{\mathrm{bpsk}}=b^{2}$. Next, we compute in $\ln \phi_{k}\left(\frac{\zeta \omega^{-k n}}{\sqrt{N}}\right)$ for a group of $1 \leq N_{\text {bpsk }} \leq N$ bins. Now, using the fact that $a_{k}$ are equi-probable we have

$$
\phi_{k}(z)=\frac{1}{2} e^{i \frac{z+\bar{z}_{b}}{2}}+\frac{1}{2} e^{-i \frac{z+\bar{z}_{b}}{2} b}=\cos \left(b \frac{z+\bar{z}}{2}\right)=\cos \left(b \Re\left\{\frac{\zeta \omega^{-k n}}{\sqrt{N}}\right\}\right)
$$

By Maclauren's series expansion we have

$$
\ln (\cos (\theta))=\sum_{j=1}^{\infty} \frac{\nu_{2 j}}{(2 j) !} \theta^{2 j},
$$

where $v_{2}=-1, v_{4}=-2, v_{6}=-16$, etc. Now,

$$
\begin{aligned}
& \sum_{k=0}^{N_{\text {bpos }}-1} \ln \left(\cos \left(b \Re\left\{\frac{\zeta \omega^{-k n}}{\sqrt{N}}\right\}\right)\right) \\
& =\sum_{k=0}^{N_{\text {bppk }}-1} \sum_{j=1}^{\infty} \frac{v_{2 j}}{(2 j) !}\left(b \Re\left[\frac{1}{\sqrt{N}} \zeta \omega^{-k n}\right]\right)^{2 j} \\
& =\sum_{j=1}^{\infty} \frac{v_{2 j}}{(2 j) !}\left(\frac{1}{2 \sqrt{N}}\right)^{2 j} \sum_{k=0}^{N_{\text {bpok }}-1} b^{2 j}\left(\zeta \omega^{-k n}+\bar{\zeta} \omega^{k n}\right)^{2 j} \\
& =\sum_{j=1}^{\infty} \frac{v_{2 j}}{(2 j) !}\left(\frac{b}{2 \sqrt{N}}\right)^{2 j} \sum_{k=0}^{N_{\text {bpak }}-1}\left[\sum_{m=0}^{2 j}\left(\begin{array}{l}
2 j \\
m
\end{array}\right)\left(\bar{\zeta} \omega^{k n}\right)^{2 j-m}\left(\zeta \omega^{-k n}\right)^{m}\right] \\
& =\sum_{j=1}^{\infty} \frac{v_{2 j}}{(2 j) !}\left(\frac{b}{2 \sqrt{N}}\right)^{2 j} \sum_{s=-j}^{j}\left(\begin{array}{c}
2 j \\
j+s
\end{array}\right) \zeta^{j+s} \bar{\zeta}-s \sum_{k=0}^{N_{\text {bppk }}-1} \omega^{-2 k n s} .
\end{aligned}
$$

Using

$$
\sum_{k=0}^{N_{\text {bpsk }}-1} \omega^{-2 k n s}= \begin{cases}N_{\text {bpsk }} & N \mid 2 n s \quad(2 n s \text { is a multiple of } N) \\ 0 & \text { otherwise }\end{cases}
$$

(27) becomes

$$
\begin{aligned}
\sum_{k=0}^{N_{\text {bpsk }}-1} \ln \cos \{\cdots\} & =\sum_{j=1}^{\infty} \frac{v_{2 j}}{(2 j) !}\left(\frac{b}{2 \sqrt{N}}\right)^{2 j} \sum_{s=-j}^{j}\left(\begin{array}{c}
2 j \\
j+s
\end{array}\right) \zeta^{j+s} \zeta^{j-s} \sum_{k=0}^{N_{\text {bpsk }}-1} \omega^{-2 k n s} \\
& =\sum_{j=1}^{\infty} \frac{v_{2 j}}{(2 j) !}\left(\frac{b}{2 \sqrt{N}}\right)^{2 j} N_{\text {bpsk }} \sum_{s=-j}^{j}\left(\begin{array}{c}
2 j \\
j+s
\end{array}\right) \zeta^{j+s} \bar{\zeta}^{j-s},
\end{aligned}
$$

where $N \mid 2 n s,-j \leq s \leq j$ and $n \in[0, \ldots, N-1]$. Next we compute the first two terms of (29), that is for $j=1,2$, as it is assumed these terms yield sufficient accuracy. The cases of $n=0, \frac{N}{4}, \frac{N}{2}, \frac{3 N}{4}$ require special attention. However, as the impact of the slightly different analytical expression for the above four cases relative to all other $n$ is negligible for practical values of $N$ (e.g., $N \geq$ 128) these cases will be neglected and treated equally as the rest of the BPSK tones.

-j $=1$ : If $n \neq 0, N / 2$ then the term $\sum_{s=-j}^{j}\left(\begin{array}{c}2 j \\ j+s\end{array}\right) \zeta^{j+s} \bar{\zeta}^{j-s}$ in (29) contains only the term $s=0$, so

$\sum_{s=-1}^{1}\left(\begin{array}{c}2 \\ 1+0\end{array}\right) \zeta^{1+0} \bar{\zeta}^{1-0}=2|\zeta|^{2}$

$-\mathbf{j}=2:$ If $n \neq 0, N / 4, N / 2,3 N / 4$ then the only possible term in the sum is $s=0$, thus the sum is

$$
\sum_{s=-2}^{2}\left(\begin{array}{c}
4 \\
2+s
\end{array}\right) \zeta^{2+s} \bar{\zeta}^{2-s}=6|\zeta|^{4}
$$

Going back to (29)and substituting the above expressions, we find the following:

$$
\begin{aligned}
\sum_{k=0}^{N_{\text {bppk }}-1} \ln \cos \{\cdots\} & =N_{\text {bpsk }}\left[\frac{\nu_{2}}{2 !} \frac{b^{2}}{2^{2} N} 2|\zeta|^{2}+\frac{\nu_{4}}{4 !} \frac{b^{4}}{2^{4} N^{2}} 6|\zeta|^{4}+\cdots\right] \\
& =N_{\text {bpsk }}\left[-\frac{b^{2}|\zeta|^{2}}{4 N}-\frac{b^{4}|\zeta|^{4}}{32 N^{2}}-\cdots\right] .
\end{aligned}
$$

- M-QAM: The QAM constellation points are drawn from the set

$a_{k} \in q \frac{[( \pm 1, \pm 3, \ldots, \pm(\sqrt{M}-1))+i \cdot( \pm 1, \pm 3, \ldots, \pm(\sqrt{M}-1))]}{\sqrt{\left(\frac{2}{3}\left(M^{2}-1\right)\right)}}$, 
i.e. the QAM constellation is symmetric and the constellation energy is $E_{\mathrm{QAM}}=q^{2}$. Symmetric QAM constellations satisfy $v_{00}=0, v_{20}=v_{02}=0$, and $v_{11}<0$. In addition, in all the symmetric cases $v_{l m}=0$ if $l+m$ is odd. We proceed by computing the expansion of $\ln \phi_{k}\left(\frac{\zeta \omega^{-k n}}{\sqrt{N}}\right)$ for a group of $1 \leq N_{\mathrm{QAM}} \leq N$ bins.

For the sake of simplicity, the expansion coefficients $v_{l m}$ here are for the unity energy constellation, hence the expansion coefficients of in $\varphi$ are $q^{l+m} v_{l m}$. For example, for QPSK of the form $a_{k_{R}}, a_{k_{I}} \in( \pm 1 / \sqrt{2})$ we have $v_{11}=-1 / 4, v_{22}=-1 / 64$, and for 16QAM of the form $a_{k_{\mathcal{R}}}, a_{k_{I}} \in( \pm 1, \pm 3) / \sqrt{10}$ we have $v_{11}=-1 / 4$ and $v_{22}=-17 / 1600$.

Then, similar to the BPSK case, we have

$$
\begin{aligned}
& \sum_{k=0}^{N_{\mathrm{QMM}}-1} \ln \phi_{k}\left(\frac{\zeta \omega^{-k n}}{\sqrt{N}}\right)=\sum_{l, m \geq 0} v_{l m} \frac{q^{l+m}}{N^{\frac{l+m}{2}}} \zeta^{l} \bar{\zeta} \sum_{k=0}^{N_{\mathrm{QMM}}-1} \omega^{-k n(l-m)} \\
& =N_{\mathrm{QAM}} \sum_{l, m: N \mid n(l-m)} v_{l m} \frac{q^{l+m}}{N^{\frac{l+m}{2}}} \zeta^{l^{2}} \bar{\zeta}^{m} \\
& =N_{\mathrm{QAM}}\left[\frac{q^{2} v_{11}|\zeta|^{2}}{N}+\frac{q^{4}}{N^{2}}\left\{v_{22}|\zeta|^{4}+\nu_{31} \zeta^{3} \bar{\zeta}+v_{13} \zeta \bar{\zeta}^{3}\right\}+\cdots\right] \text {. }
\end{aligned}
$$

We next decompose the symbols vector $\boldsymbol{a}$ of length $N$ into three groups:

- $N_{B}$ groups of BPSK symbols. The symbols of each group, of size $N_{r}$, are drawn from a constellation of energy $E_{r}=b_{r}^{2}$ for $1 \leq r \leq N_{B}$.

- $N_{Q}$ groups of QAM symbols. The symbols of each group, of size $N_{s}$, are drawn from a constellation of size $M_{s}$ (that is, the coefficients $v_{l m}$ are constellation dependent and are denoted as $v_{s, l m}$ ) and energy $E_{s}=q_{s}^{2}$ for $1 \leq s \leq N_{Q}$.

- $N_{Z}$ zero tones.

Obviously, $N_{B}+N_{Q}+N_{Z}=N$.

Following(24),the expansions of In $\varphi_{k}$ of all groups are summed:

$$
\begin{aligned}
& \sum_{k=0}^{N-1} \ln \phi_{k}\left(\frac{\zeta \omega^{k h n}}{\sqrt{N}}\right)=\sum_{r=1}^{N_{n}}\left(-\left(-\frac{b_{t}^{2} N_{T}|\zeta|^{2}}{4 N}-\frac{b_{t}^{4} N_{T}|\zeta|^{4}}{32 N^{2}}-\cdots\right)\right. \\
& +\sum_{s=1}^{N_{Q}}\left(\frac{q_{s}^{2} N_{s} v_{s, 11}|\zeta|^{2}}{N}+\frac{q_{s}^{4} N_{s}}{N^{2}}\left\{v_{s, 22}|\zeta|^{4}+v_{s, 31} \zeta^{3} \bar{\zeta}+v_{s, 13} \zeta^{3} \xi^{3}\right\}+\cdots\right)
\end{aligned}
$$

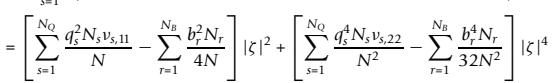

$$
\begin{aligned}
& +\sum_{s=1}^{N_{Q}} \frac{q_{S}^{4} N_{s}}{N^{2}}\left[v_{s, 3}, 3 \zeta^{3} \bar{\zeta}+v_{s, 13} \zeta \bar{\zeta}^{3}\right]+\cdots
\end{aligned}
$$

Denoting $\quad \mu_{1}=\left[\sum_{s=1}^{N_{Q}} \frac{q_{s}^{2} N_{s} v_{s, 11}}{N}-\sum_{r=1}^{N_{B}} \frac{b_{r}^{2} N_{r}}{4 N}\right]$ and $\mu_{2}=\left[\sum_{s=1}^{N_{Q}} \frac{q_{s}^{4} N_{s} v_{s, 22}}{N^{2}}-\sum_{r=1}^{N_{B}} \frac{b_{r}^{4} N_{r}}{32 N^{2}}\right]$ we have

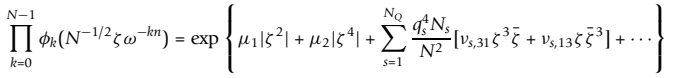

$$
\begin{aligned}
& =\exp \left\{\mu_{1}|\zeta|^{2} \exp \left\{\mu_{2}|\zeta|^{4}+\sum_{j=1}^{N_{0}} \frac{q_{N}^{4} N_{s}}{N^{2}}\left|v_{s, 3}\right| \zeta^{3} \xi+v_{s, 13} \xi^{3}\right]+\cdots\right\}
\end{aligned}
$$

Now, using $e^{x}=1+x+\ldots$ we have

$$
\left.\left.\prod_{k=0}^{N-1} \phi_{k}\left(N^{-1 / 2} \zeta \omega^{-k n}\right)=\exp \left\{\mu_{1}|\zeta|^{2}\right\} \cdot\left[1+\mu_{2}|\zeta|^{4}+\sum_{\xi=1}^{N_{0}} \frac{q_{s}^{4} N_{s}}{N^{2}} \mid v_{s, 3} \xi_{s} \zeta^{3} \bar{\xi}+v_{s, 13} \zeta \zeta^{3}\right\}\right]+\cdots\right] .
$$

Following (20), we multiply (37) by $\frac{1}{2 \pi} J_{0}(r|\zeta|)$ and integrate over $\mathbb{R}^{2}$. First, we pass to polar coordinates $u, \theta$ (i.e. $\zeta=u \exp (i \theta)$ ), and observe that all the terms $\zeta^{\bar{\zeta}^{m}}$ with $l \neq m$ vanish (since the integral of $\cos ((l-m) \theta)$ is zero). Therefore, we are left with

$$
\int_{0}^{\infty} J_{0}(r u) \exp \left\{\mu_{1} u^{2}\right\}\left\{u+\mu_{2} u^{5}+\cdots\right\} d u .
$$

Using [14,(6.631)] we arrive at

$$
\left.\left.\int_{0}^{\infty} J_{0}(r u) \exp \left\{\mu_{1} u^{2}\right\}\right] u+\mu_{2} u^{5}\right] d u=-\frac{1}{2 \mu_{1}}{ }_{1} F_{1}\left(1,1, \frac{r^{2}}{4 \mu_{1}}\right)-\frac{\mu_{2}}{\mu_{1}^{1} F_{1}}\left(3,1, \frac{r^{2}}{4 \mu_{1}}\right) .
$$

Using the identities ${ }_{1} F_{1}(1,1, z)=e^{z}$ and ${ }_{1} F_{1}(3,1, z)=e^{z}\left(1+2 z+z^{2} / 2\right)$ and summing up $N$ times (20), we get

$$
\sum_{n=0}^{N-1} \mathbb{E}\left\{f\left(x_{n \times} x_{n 1}\right)\right\}=N \int_{0}^{\infty} r f(r) \exp \left(\frac{r^{2}}{4 \mu_{1}}\right)\left\{-\frac{1}{2 \mu_{1}}-\frac{\mu_{2}}{\mu_{1}^{3}}\left(1+\frac{r^{2}}{2 \mu_{1}}+\frac{r^{4}}{32 \mu_{1}}\right)-\cdots\right\} d r .
$$

Denoting $\tilde{\mu}_{1}=N \mu_{1}$ and $\tilde{\mu}_{2}=N^{2} \mu_{2}$, (40) can be rewritten as

$$
\begin{aligned}
\sum_{n=0}^{N-1} \mathbb{E}\left\{f\left(x_{n_{\alpha}} x_{n_{n}}\right)\right\}= & N^{2}\left\{\left[-\int_{0}^{\infty} r f(r) \exp \left(\frac{r^{2}}{4 \mu_{1}}\right)\left(\frac{1}{2 \tilde{\mu}_{1}}+\frac{\tilde{\mu}_{2}}{\widetilde{\mu}_{1}^{3}}\right) d r\right]\right. \\
& \left.+N\left[-\int_{0}^{\infty} r f(r) \exp \left(\frac{r^{2}}{4 \mu_{1}}\right)\left(\frac{\tilde{\mu}_{2}}{2 \tilde{\mu}_{1}^{4}} t^{2}+\frac{\tilde{\mu}_{2}}{32 \tilde{\mu}_{1}^{5}} r^{4}\right) d r\right]+\cdots\right\},
\end{aligned}
$$

and following (9) we have

$$
m_{0}(c)=-\int_{0}^{\infty} r f(r) \exp \left(\frac{r^{2}}{4 \mu_{1}}\right)\left(\frac{1}{2 \tilde{\mu}_{1}}+\frac{\tilde{\mu}_{2}}{\tilde{\mu}_{1}^{3}}\right) d r
$$

and

$$
m_{1}(c)=-\int_{0}^{\infty} r f(r) \exp \left(\frac{r^{2}}{4 \mu_{1}}\right)\left(\frac{\tilde{\mu}_{2}}{2 \tilde{\mu}_{1}^{4}} r^{2}+\frac{\tilde{\mu}_{2}}{32 \tilde{\mu}_{1}^{r}} r^{4}\right) d r .
$$

\section{Abbreviations}

CLT: central limit theorem; EVM: error vector magnitude; HPA: high-power amplifier; MC: multi-carrier; PAPR: peak-to-average power ratio.

\section{Acknowledgement}

The authors would like to thank Eyal Verbin for his contribution to this work.

\section{Competing interests}

The authors declare that they have no competing interests.

Received: 27 November 2010 Accepted: 8 August 2011

Published: 8 August 2011

\section{References}

1. S Litsyn, Peak Power Control in Multicarrier Communications (Cambridge University Press, Cambridge, 2007) 
2. SH Han, JH Lee, An overview of peak-to-average power ratio reduction techniques for multicarrier transmission. IEEE Wireless Commun Mag. 12, 56-65 (2005). doi:10.1109/MWC.2005.1421929

3. T Jiang, $Y$ Wu, An overview: peak-to-average power ratio reduction techniques for OFDM signals. IEEE Trans Broadcast. 54(2), 257-268 (2008)

4. J Tellado, Multicarrier Modulation with Low PAR (Kluwer Academic Publishers, Dordrecht, 2000)

5. H Ochiai, H Imai, Performance analysis of deliberately clipped ofdm signals. IEEE Trans Commun. 50(1), 89-101 (2002). doi:10.1109/26.975762

6. F Peng, WE Ryan, On the capacity of clipped ofdm channels, in ISIT2006, Seattle, USA, 9-14 July 2006 (2006)

7. R Raich, H Qian, G Zhou, Optimization of SNDR for amplitude-limited nonlinearities. IEEE Trans Commun. 53(11), 1964-1972 (2005). doi:10.1109/ TCOMM.2005.857141

8. A Bahai, M Singh, A Goldsmith, B Saltzberg, A new approach for evaluating clipping distortion in multicarrier systems. IEEE J Sel Areas Commun. 20(5), 1037-1046 (2002). doi:10.1109/JSAC.2002.1007384

9. H Nikopour, S Jamali, On the performance of OFDM systems over a Cartesian clipping channel: a theoretical approach. IEEE Trans Wireless Commun. 3(6), 2083-2096 (2004). doi:10.1109/TWC.2004.837425

10. B Cutler, Effects of physical layer impairments on ofdm systems. RF Design, 36-44 (May 2002)

11. IEEE standard for local and metropolitan area networks, part 16: Air interface for fixed broadband wireless access systems, IEEE, Technical Report (2004)

12. I Kotzer, S Har-Nevo, S Sodin, S Litsyn, An analytical approach to the calculation of EVM in clipped multi-carrier signals (in press)

13. C Rapp, Effects of hpa-nonlinearity on a 4-dpsk/ofdm-signal for a digital sound broadcasting system. in Second European Conference on Satellite Communications, 22-24.10.91, Liege, Belgium, 179-184 (1991)

14. I Gradshteyn, I Ryzhik, Table of Integrals, Series and Products (Elsevier, Amsterdam, 2007)

doi:10.1186/1687-6180-2011-36

Cite this article as: Kotzer and Litsyn: On the EVM computation of arbitrary clipped multi-carrier signals. EURASIP Journal on Advances in Signal Processing 2011 2011:36.

\section{Submit your manuscript to a SpringerOpen ${ }^{\circ}$ journal and benefit from:}

- Convenient online submission

- Rigorous peer review

- Immediate publication on acceptance

- Open access: articles freely available online

- High visibility within the field

- Retaining the copyright to your article

Submit your next manuscript at $\gg$ springeropen.com 